\title{
First-principles total-energy calculation of gallium nitride
}

\author{
B. J. Min, C. T. Chan, and K. M. Ho \\ Ames Laboratory, Iowa State University, Ames, Iowa 50011 \\ and Department of Physics and Astronomy, Iowa State University, Ames, Iowa 50011
}

(Received 20 March 1991; revised manuscript received 28 August 1991)

\begin{abstract}
A first-principles total-energy calculation is performed on gallium nitride $(\mathrm{GaN})$. The equilibrium lattice parameters, the bulk modulus, and the cohesive energy of $\mathrm{GaN}$ in the wurtzite structure is calculated and compared with experimental values. In our calculation, the ground state of $\mathrm{GaN}$ is a zinc-blende structure, and the difference between these two phases is around $1.4 \mathrm{mRy}$.
\end{abstract}

\section{INTRODUCTION}

Gallium nitride $(\mathrm{GaN})$ is a tetrahedrally coordinated III-V compound semiconductor which has the hexagonal wurtzite structure with a wide band gap of $3.5 \mathrm{eV}$ at 300 $\mathrm{K}$. It is reported that a simple-metal-insulator- $n$-typeGaN diode produces blue electroluminescence. ${ }^{1}$ For this reason it has been studied as a material suitable for an effective optoelectronic device such as laser and light emitting diode, but the technical difficulties are yet to be surmounted.

The major difficulty has been that the concentration of free electrons is too large $\left(10^{18}-10^{20} \mathrm{~cm}^{-3}\right)$ for the fabrication of a p-type crystal to be successful. It is believed that nitrogen vacancies are the source of free electrons rather than some unknown donor impurities. ${ }^{2}$ The growth of a GaN crystal has had other difficulties, too. The GaN substrate on sapphire has different lattice constants and luminescence characteristics depending on the direction of growth. ${ }^{3}$ Also there is a report about an unusually large variation of the lattice constants depending on the growth conditions, such as growth rate, lattice defects, and foreign impurities. ${ }^{4,5}$

Thus it is of importance to understand the energetics of the GaN crystal. A first-principles total-energy calculation can provide useful information for studying the energetics of a solid-state system. Without introducing empirical parameters, it provides a good description of the equilibrium properties such as lattice structure, lattice constant, cohesive energy, and bulk modulus. Such input from first-principles calculations can be helpful for understanding the nature of the current problem in crystal growth.

We also present the electronic band structure of GaN from our calculations. Currently there are band calculations that do not agree with each other ${ }^{6-9}$ about the nature of the band gap. Although it is well known that local density approximation ${ }^{10}$ (LDA) calculations usually underestimate the size of the band gap, it is accurate enough for one to determine whether the band gap is direct or not.

\section{METHOD OF CALCULATION}

The calculations are performed using the densityfunctional method within the local-density approximation. Norm-conserving pseudopotentials are used both for $\mathrm{Ga}$ and $\mathrm{N}$. The pseudopotentials were generated according to the Hamann, Schlüter, and Chiang scheme. ${ }^{11}$ One of the advantages of this scheme is that the pseudopotential is determined entirely from $a b$ initio atomic calculations, and hence no empirical parameters are needed. In this scheme, the pseudo-wave-functions are constructed such that they coincide with the all-electron atomic

TABLE I. The Ga pseudopotential eigenvalues and the excitation energies compared with the allelectron self-consistent calculation results (in parentheses).

\begin{tabular}{lcccc}
\hline \hline & $E_{4 s}$ & $E_{4 p}$ & $E_{4 d}$ & Excitation energy \\
\hline $4 s^{2} 4 p^{1}$ & -0.675 & -0.206 & & 0.000 \\
& $(-0.679)$ & $(-0.208)$ & & $(0.000)$ \\
$4 s^{1} 4 p^{2}$ & -0.758 & -0.263 & & 0.482 \\
& $(-0.760)$ & $(-0.265)$ & & $(0.484)$ \\
$4 d^{3}$ & -0.830 & -0.311 & & 0.990 \\
& $(-0.830)$ & $(-0.311)$ & & $(0.992)$ \\
$4 s^{0.75} 4 p^{0.25} 4 d^{0.25}$ & -1.861 & -1.249 & -0.563 & 2.030 \\
$4 s^{0.5} 4 p^{0.5} 4 d^{0.25}$ & $(-1.850)$ & $(-1.244)$ & $(-0.564)$ & $(2.029)$ \\
$4 s^{0.5} 4 p^{0.5} 4 d^{0.25}$ & -1.880 & -1.264 & -0.571 & 2.184 \\
& $(-1.868)$ & $(-1.259)$ & $(-0.572)$ & $(2.181)$ \\
\hline
\end{tabular}




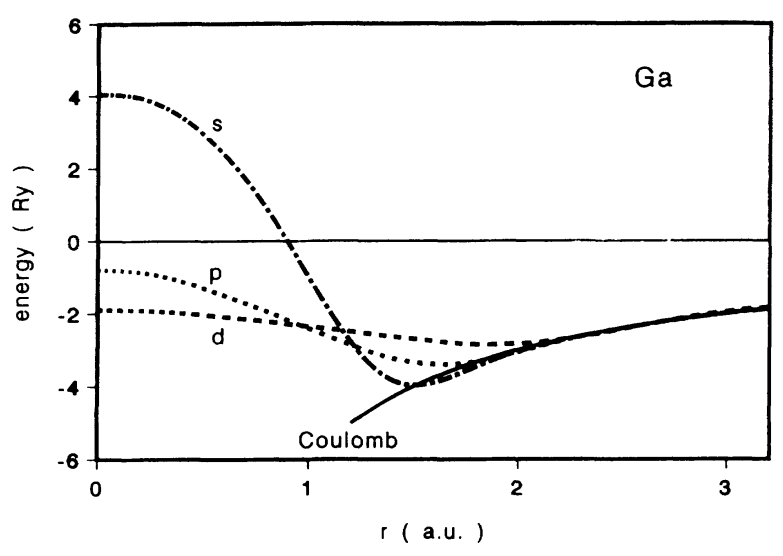

FIG. 1. The angular-momentum-dependent pseudopotential of Ga shown with the Coulomb potential (dotted line).

wave functions outside some chosen core radius, while maintaining the same eigenvalues. The all-electron atomic wave functions and eigenvalues are obtained from a separate atomic calculation.

For gallium, we solved the all-electron relativistic Dirac equation to get the wave functions and the eigenvalues. We tested several different configurations, both ionized and neutral, so as to choose the reference configuration for generation of the pseudopotential. In the test of the pseudopotential, we considered atomic configurations with excitation energies less than $1 \mathrm{Ry}$ and checked if the pseudoeigenvalues and the pseudoatom excitation energies are in good agreement with the allelectron atomic values. We found that the transferability of the pseudopotential we generated was not very satisfactory. This can be traced to the fact that there is nontrivial core and valence-electron overlap. The partial core correction scheme according to Louie, Froyen, and Cohen ${ }^{12}$ is found to improve the transferability substantially. The pseudopotential built upon the ionic configuration $4 s^{1.5} 4 p^{0.25} 4 d^{0.25}$ has the best transferability among the tested configurations. The eigenvalues and the excitation energies given by the pseudopotential are shown in Table I. The angular-momentum-dependent pseudopotential is plotted in Fig. 1. The pseudopotential

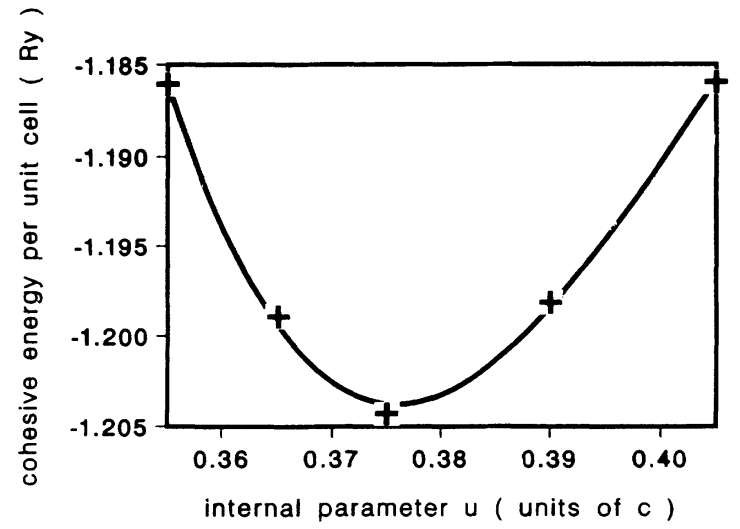

FIG. 2. The total energy of GaN per unit cell vs sublattice displacement $u$ (in units of $c$ ).

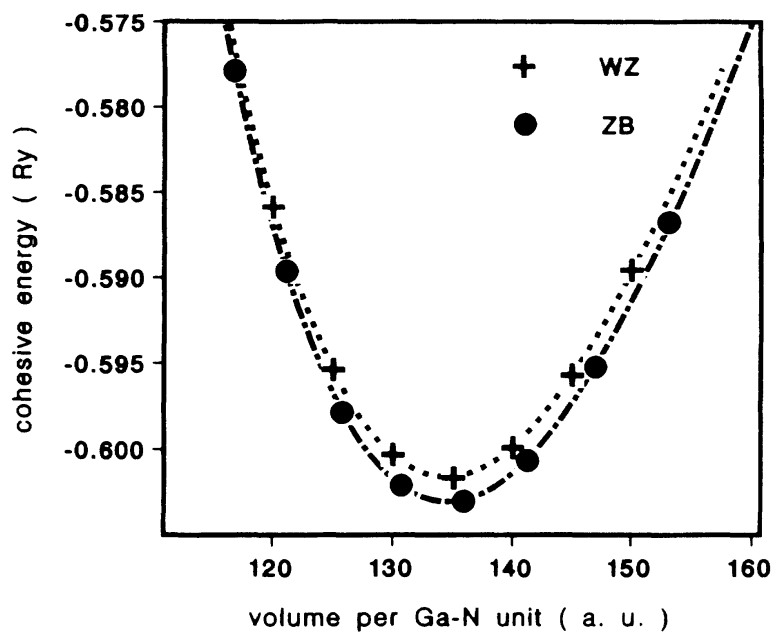

FIG. 3. The total energy of GaN per unit cell vs cell volume (a.u.) for the wurtzite (WZ) and the zinc-blende (ZB) structure.

for nitrogen is taken from Bachelet, Hamann, and Schlüter. ${ }^{13}$

The total-energy and the electronic structure calculations of $\mathrm{GaN}$ using pseudopotentials is complicated by the sharp $s$ and $p$ states of nitrogen, which would require a large number of plane waves for a convergent expansion of the Bloch wave functions. This problem is alleviated by the use of a mixed-basis set, ${ }^{14}$ which consists of the Bloch sums of the local orbitals and the plane waves up to a cutoff energy of $14 \mathrm{Ry}$. The local orbitals $\phi_{s}$ and $\phi_{p}$ for the nitrogen $s$ and $p$ states have been constructed from the eigenfunctions $\psi_{2 s}$ and $\psi_{2 p}$ of the nitrogen pseudopotential. For the $s$ state, the local orbital is constructed by

$$
\phi_{s}(\mathbf{r})=\left\{\begin{array}{l}
N\left[\psi_{2 s}(r)-\alpha j_{0}(q r)\right], \quad r<r_{c} \\
0, \quad r>r_{c},
\end{array}\right.
$$

where $N$ is a normalization constant, and $\alpha$ and $q$ are determined such that

$$
\begin{aligned}
& \left.\psi_{s}(r)\right|_{r=r_{c}}=\left.\alpha j_{0}(q r)\right|_{r=r_{c}}, \\
& \left.\psi_{s}^{\prime}(r)\right|_{r=r_{c}}=\left.\alpha \frac{d}{d r} j_{0}(q r)\right|_{r=r_{c}} .
\end{aligned}
$$

TABLE II. Comparison of theoretical and experimental $c$ and $a$ values of $\mathrm{GaN}$ in the wurtzite structure.

\begin{tabular}{llll}
\hline \hline & $a$ (a.u.) & $c$ (a.u.) & $c / a$ \\
\hline Calculation & 5.75 & 9.39 & 1.634 \\
Experiment $^{\mathrm{a}}$ & 6.030 & 9.796 & 1.625 \\
Experiment $^{\mathrm{b}}$ & 5.963 & 9.861 & $1.653^{\text {d }}$ \\
& 6.006 & 9.777 & $1.628^{\mathrm{e}}$ \\
Experiment $^{\mathrm{c}}$ & 6.028 & 9.806 & 1.627 \\
\hline
\end{tabular}

${ }^{\text {a Reference } 4 .}$.

${ }^{\mathrm{b}}$ Reference 3.

${ }^{\mathrm{c}}$ Reference 19.

${ }^{\mathrm{d}} 10$ layers of $\mathrm{GaN}$ deposited onto (10, 2$) \alpha-\mathrm{Al}_{2} \mathrm{O}_{3}$.

${ }^{e} 28$ layers of $\mathrm{GaN}$ deposited onto (0001) $\alpha-\mathrm{Al}_{2} \mathrm{O}_{3}$. 
The radial part of the $p$-state local orbital $\phi_{p}$ is constructed by

$$
\phi_{p}(r)=\left\{\begin{array}{l}
N\left\{1-\exp \left[-\beta\left(r_{c}-r\right)^{2}\right]\right\} \psi_{2 p}(r), \quad r<r_{c} \\
0, \quad r>r_{c}
\end{array}\right.
$$

where $N$ is a normalization constant, $r_{c}=1.6$ a.u. is the cutoff radius, and $\beta=1.61$ a.u. has been used. We have observed that taking a good basis set is crucial for obtaining a good result and also for the reduction of noise. Since the pseudopotential for nitrogen is very sharp, it is expedient to employ the real-space charge symmetrization scheme $e^{15}$ rather than symmetrizing the Fourier components of the charge, which would require a tremendous number of plane waves. We observed that the wurtzite structure was lower in energy than the zinc-blende structure when the basis set was poor. However, as we improve the basis set by adopting sharp local orbitals and by increasing the number of plane waves, the order reverses, and the zinc-blende structure becomes lower in energy.

The momentum space expression of the total energy ${ }^{16}$ is used to calculate the total energy. The charge and the electronic screening potential are found iteratively until the total energy converges within $10^{-6} \mathrm{Ry}$ and the screening potential within $10^{-5} \mathrm{Ry}$. The eigenvalues are sampled at $30 \mathbf{k}$ points within an irreducible wedge of the Brillouin zone (IBZ) in the case of wurtzite structure. In the zinc-blende structure, we have sampled $60 \mathrm{k}$ points.

\section{RESULT AND DISCUSSION}

First we fixed the $c / a$ ratio of the wurtzite structure at the ideal value of $\left(\frac{8}{3}\right)^{1 / 2}$, and performed the firstprinciples total-energy calculations to determine the atomic volume. The calculated equilibrium volume is 270 a.u. per unit cell which contains two $\mathrm{Ga}$ atoms and two $\mathrm{N}$ atoms.

Then the total-energy calculation is repeated for different $c / a$ ratios with the volume fixed at 270 a.u. The $c / a$ ratio is found to be 1.634 , which is very close to the ideal ratio.

The internal parameter $u$, the relative displacement of the $N$ sublattice with respect to the Ga sublattice along the $c$ direction, is determined at the above equilibrium volume and $c / a$ ratio. By minimizing the total energy of the system, we have found that this sublattice displacement $u$ is $0.375 c$ and is again very close to the ideal value of $3 c / 8$ (Fig. 2). The frequency of the $A_{1}-\mathrm{TO}(\Gamma)$-phonon mode is calculated to be $640 \mathrm{~cm}^{-1}$ from the second derivative of the curve in Fig. 2. The frequency of this mode has been measured to be $533 \mathrm{~cm}^{-1}$ by Raman spectroscopy. ${ }^{17}$

The energy versus volume calculation is then repeated with $c / a=1.634$. The equilibrium lattice constant, the cohesive energy, and the bulk modulus are obtained by fitting to the universal binding curve ${ }^{18}$

$$
E(a)=\Delta E E^{*}\left(a^{*}\right),
$$

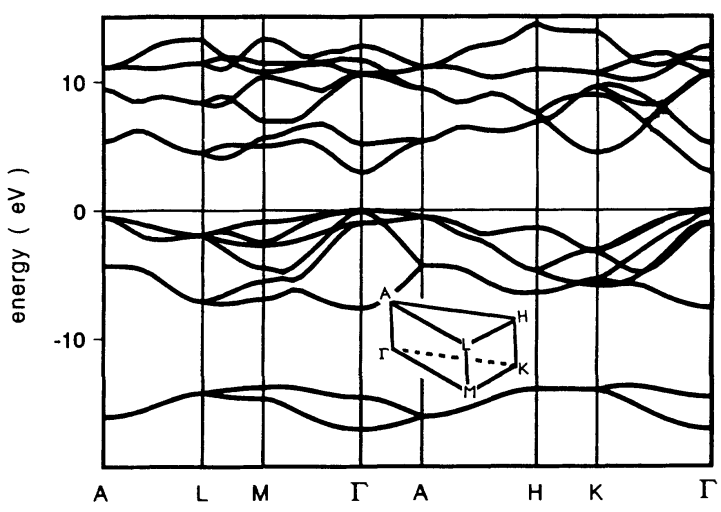

FIG. 4. The energy-band structure of $\mathrm{GaN}$ in wurtzite structure.

where $a^{*}=\left(a-a_{m}\right) / l, \quad E^{*}\left(a^{*}\right)=\left(1+a^{*}\right) \exp \left(-a^{*}\right)$, with three parameters $a_{m}, \Delta E$, and $l$. The error in the fitting is less than $0.1 \mathrm{mRy}$. We get $134 \mathrm{a}$.u. for the equilibrium volume per Ga-N unit, 2.4 Mbar for the bulk modulus, and $0.602 \mathrm{Ry}$ for the cohesive energy per one Ga-N unit (Fig. 3).

The comparison of the lattice constants with the experimental values are summarized in Table II. As can be seen in Table II, experimental values are generally larger than our calculation. Furthermore, the dependence of lattice constants on the growth conditions suggest that it is very difficult experimentally to grow a good GaN crystal.

The electronic band structure of $\mathrm{GaN}$ in wurtzite structure is plotted in Fig. 4. It is important for the fabrication of an optoelectronic device that the gap is direct. There have been other electronic band calculations of $\mathrm{GaN}$, but as to the nature and the magnitude of the energy gap, there is a wide variation depending on the methods used. The model pseudopotential calculation by Grinyaev, Malakhov, and Chaldshev ${ }^{6}$ predicts an indirect energy gap of $3.0 \mathrm{eV}$. The empirical pseudopotential calculation by Bloom et al. ${ }^{7}$ predicts a direct gap of $3.6 \mathrm{eV}$. The orthogonalized linear combination of atomic orbital (LCAO) calculation by Huang and $\mathrm{Ching}^{9}$ gives a direct

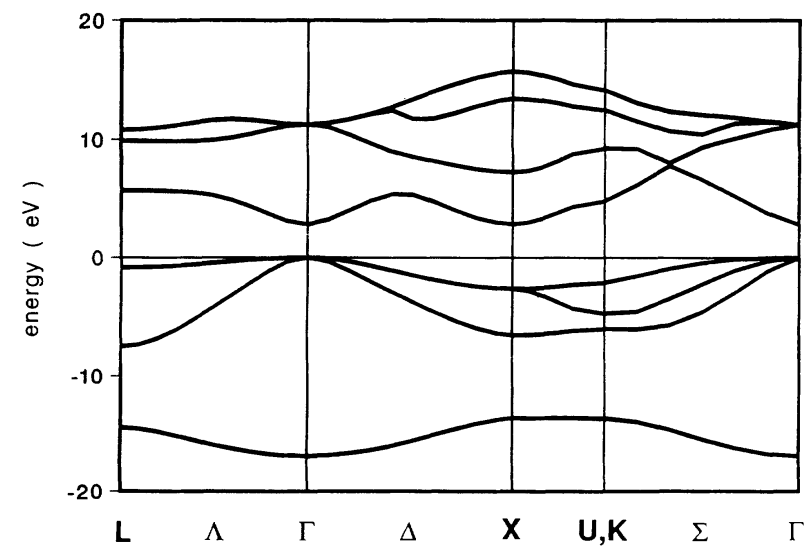

FIG. 5. The energy-band structure of $\mathrm{GaN}$ in zinc-blende structure. 
gap of $3.5 \mathrm{eV}$. Our result has a direct band gap of $3.0 \mathrm{eV}$ at the $\Gamma$ point. As it is widely known that the LDA calculations underestimate the size of the energy gap, we can expect the real energy gap to be larger than our value, though the energy gap would remain direct.

Since it is very difficult to have a good GaN crystal, we have investigated the other possible structure of $\mathrm{GaN}$ crystal by the total-energy calculation, i.e., the zincblende structure. In this calculation $60 \mathrm{k}$ points are sampled from the irreducible part of the Brillouin zone. The result is again fitted to the universal binding curve to determine the cohesive energy, atomic volume, and the bulk modulus. We have $0.603 \mathrm{Ry}$ as the cohesive energy per primitive unit cell, 134 a.u. as the volume per primitive unit cell, and $2.4 \mathrm{Mbar}$ as the bulk modulus. The error in the fitting is less than $0.2 \mathrm{mRy}$. The zinc-blende structure appears to be ground state at zero temperature according to our calculation. However, the cohesive energy, the equilibrium volume, and the bulk modulus are quite close in the zinc-blende and wurtzite structures. The cohesive energies of the two structures differ by only $1.4 \mathrm{mRy}$ per one Ga-N unit. As we vary the plane-wave cutoff, the zinc-blende structure remains lower in energy than the wurzite structure. The difference in their total energies per $\mathrm{Ga}-\mathrm{N}$ unit is $1.4,1.6$, and $1.3 \mathrm{mRy}$, at cutoff energies of 14,16 , and $18 \mathrm{Ry}$, respectively. Since this energy difference is very small and can be readily surmounted by thermal energy at room temperature, it would be interesting to consider the possibility of a phase transition between these two phases. The band structure of $\mathrm{GaN}$ in the zinc-blende structure (Fig. 5) shows that it has a direct band gap $E_{g}=2.8 \mathrm{eV}$ at the $\Gamma$ point.

To summarize, we have performed a first-principles total-energy calculation for the $\mathrm{GaN}$ crystal and made a prediction of equilibrium lattice constants, cohesive energy, and the bulk modulus for two possible crystal structures. Also the frequency of the $A_{1}-\mathrm{TO}(\Gamma)$-phonon mode in the wurtzite structure compares well with the experiment. It is predicted that the zinc-blende structure is lower in energy than the wurtzite structure, but the energy between the two structures is so close that the effects of substrates and defects have to be considered to determine which crystal structure will be preferred under various crystal-growth conditions.

\section{ACKNOWLEDGMENTS}

The authors wish to express thanks to Dr. Bruce Harmon for many valuable suggestions and for carefully reading the manuscript. This work was supported by the National Science Foundation under Grant No. DMR8819379 and in part by the Director of Energy Research, Office of Basic Energy Sciences. Ames Laboratory is operated for the U.S. Department of Energy by Iowa State University under Contract No. W-7405-ENG-82.
${ }^{1}$ J. I. Pankove, E. A. Miller, and J. E. Berkeyheiser, J. Lumin. 5, 84 (1972).

2J. Karpiński, J. Jun, and S. Porowski, J. Cryst. Growth 66, 1 (1984).

${ }^{3}$ I. F. Chetverikova, M. V. Chukichev, and L. N. Rastorguev, Izv. Akad. Nauk SSSR Neorg. Mater. 22, 63 (1986) [Inorg. Matter. (USSR) 22, 53 (1986)].

${ }^{4}$ W. Seifert, H.-G. Brühl, and G. Fitzel, Phys. Status Solidi A 61, 493 (1980).

${ }^{5}$ O. Lagerstedt and B. Monemar, Phys. Rev. B 19, 3064 (1979).

${ }^{6}$ S. N. Grinyaev, V. Ya. Malakhov, and V. A. Chaldyshev, Izv. Vyssh. Uchebn. Zaved Fiz. 28, 3 (1985). [Sov. Phys. J. 28, 251 (1985)].

${ }^{7}$ S. Bloom, G. Harbeke, E. Meier, and I. B. Ortenburger, Phys. Status Solidi B 66, 161 (1974).

${ }^{8}$ D. Jones and A. H. Lettington, Solid State Commun. 11, 701 (1972).

${ }^{9}$ M. Huang and W. Y. Ching, J. Phys. Chem. Solids 46, 977 (1985).

${ }^{10}$ P. Hohenberg and W. Kohn, Phys. Rev. 136, B864 (1964); W.
Kohn and J. Sham, ibid. 140, A1133 (1965).

${ }^{11}$ D. R. Hamann, M. Schlüter, and C. Chiang, Phys. Rev. Lett. 43, 1494 (1979).

${ }^{12}$ S. G. Louie, S. Froyen, and M. L. Cohen, Phys. Rev. B 26, 1738 (1982).

${ }^{13}$ G. B. Bachelet, D. R. Hamann, and M. Schlüter, Phys. Rev. B 26, 4199 (1982).

${ }^{14}$ S. G. Louie, K.-M. Ho, and M. L. Cohen, Phys. Rev. B 19, 1774 (1979).

${ }^{15}$ C. Elsässer, N. Takeuchi, K. M. Ho, C. T. Chan, P. Braun, and M. Fähnle, J. Phys. Condens. Matter 2, 4371 (1990).

${ }^{16} \mathrm{~J}$. Ihm, A. Zunger, and M. L. Cohen, J. Phys. C 12, 4409 (1979).

${ }^{17}$ D. D. Manchon, A. S. Barker, J. P. Dean, and R. B. Zetterstrom, Solid State Commun. 8, 1227 (1970).

18 J. H. Rose, J. Ferrante, and J. R. Smith, Phys. Rev. Lett. 47, 675 (1981).

${ }^{19}$ H. Schulz and K. H. Thiemann, Solid State Commun. 23, 815 (1977). 